\title{
Sparse Reconstruction and Geo-Registration of Site Photographs for As-Built Construction Representation and Automatic Progress Data Collection
}

\author{
Mani Golparvar-Fard ${ }^{1}$, Feniosky Peña-Mora ${ }^{2}$ and Silvio Savarese ${ }^{3}$
}

1 William E. O'Neil Pre-Doctoral Candidate in Construction Management and Master of Computer Science Student, University of Illinois, Urbana-Champaign, 205 N. Mathews Ave., Urbana, IL 61801; PH (217) 3332071; FAX (217) 265-8039; email: mgolpar2@illinois.edu

${ }^{2}$ E.W. and J.M. Gutgsell Endowed Professor in Construction Management and Information Technology, Civil and Environmental Engineering, University of Illinois, Urbana-Champaign, 205 N. Mathews Ave., Urbana, IL 61801; PH (217) 244-0187; FAX (217) 265-8039; email: feniosky@illinois.edu

3 Assistant Professor, Electrical and Computer Engineering, University of Michigan, Ann Arbor, MI 481092122; PH (734) 647-8136; FAX (217) 265-8039; e-mail: silvio@eecs.umich.edu

\begin{abstract}
Most of the current techniques for automating progress data collection promise to eliminate laborintensive tasks associated with manual data collection. A drawback is the necessity to add additional steps to be performed before, during, or after utilization of such technologies. Working with such featureless data and without having semantic information of the scene, geometric-reasoning is problematic and induces estimation errors. In this paper application of unordered daily progress photograph logs, available on any job site, as a data collection technique is explored. In our proposed approach, a sparse 3D geometric scene of a construction site is reconstructed and photographs are geo-registered. This allows project managers to remotely explore as-built scene and geo-registered site photographs at different stages of progress, minimize their travel time, perform remote as-built analysis and use the proposed system as a tool for contractor coordination purposes. Furthermore, the point cloud allows the planned model to be registered with the asbuilt scene, in turn supporting development of the automatic 3D recognition technique and quantification of as-built progression from the geo-registered images. We present our results on two ongoing construction projects and further discuss technical issues on developing and implementing this technology for automation and visualization of as-built construction.
\end{abstract}

\section{Introduction}

In today's economy, construction companies are seeking new ways to streamline their work processes to reduce project durations and costs. The reasons are simple: owners need to minimize time and cost for their services or marketing products and thus need to reduce the delivery time for facilities that provide such services or products. Along the same line, contractors are faced with intense competition, tight market constraints and slim profit margins. These situations motivate contractors to detect actual or potential delays and cost overruns in field activities as early as possible. Systematic and comprehensive tracking and monitoring of construction performance, workforce productivity, site layout and quality provides managers with an opportunity to detect such delays and overruns, initiate remedial actions and increase the chance of controlling their impacts.

Despite the importance, current practice of data collection experiences several process inefficiencies. Every day, superintendents and field engineers must collect and extract extensive amount of as-built data (Navon and Sacks 2007) which in turn may cause human-errors and induce error in the manually collected data (observed by authors). The excessive load of the required work usually makes monitoring task nonsystematic and leaves it to be based largely upon judgments derived from past experiences. This may create a tendency to let project plan inputs be used as performance measures which in turn affects quality of the results (Meredith and Mantel 2003). Some of the currently used techniques such as cost-based monitoring may create a time-lag between the time that actual progress is reported and the time that progress is actually obtained. Also site activities are more numerous than what plans usually describe. Consistent changes on the job site including location of construction equipment, workforce, materials and the work sequences usually not included in the original plans are left to be noted in text or chart forms (Shih et al. 2004). These 
recording and reporting forms may increase the time required to describe and explain the as-built situation, consistently changing layouts and constructability issues in coordination meetings as well as delaying the decision-making process (Golparvar-Fard et al. 2006). In summary, with current data collection, analysis and reporting methods it may not be easy to clearly and quickly understand the progress situation. Functions that enable automatic digital recording, identification and reporting of the as-built construction site will be useful.

Most of the current technologies for automating data collection (such as laser scanners, Radio Frequency Identification (RFID) tags, Global Positioning Systems (GPS), Wireless Fidelity (Wi-Fi) and Ultra WideBands (UWB) sensors are promising if one wishes to eliminate labor-intensive and non-value adding tasks associated with manual site data collection. However, a drawback in application of these technologies is the necessity to add additional steps needed to be performed before, during, or after utilization of such technologies at a construction site (Kiziltas et al. 2008). For instance, by using laser scanners, only Cartesian coordinate information of the scanned scene could be retrieved. Working with such featureless data and without any semantic information of the scene, geometric reasoning based on this data is problematic and induces estimation errors.

In this paper, application of unordered daily progress photograph logs - which is currently available on almost any construction site - as a data collection technique is explored. Nowadays site photographs are becoming valuable sources of accurate project information (Soibelman et al. 2008). It is very common among all parties involved in projects (from construction managers to subcontractors and clients to architects) to take digital photographs from construction sites to create a complete progress photo-log and utilize the log for coordination and communication purposes as well as collecting them as supplementary documents for potential claims. Cameras can cover significant areas of a construction site, especially if outfitted with zoom lenses. They have the capability of providing positioning information about multiple construction entities concurrently. All of these facts indicate that project photographs have evolved into a significant and irreplaceable part of project documentation and thus providing solid participations for their usage as visual, real-time as well as easy to obtain, low-price data capturing technology which does not need any expertise. The availability of such rich imagery of large parts seen under different viewing conditions motivated this study to see how based on this valuable dataset, digital representation of the as-built site can be generated, allowing progress to be tracked and workspace logistics, constructability, quality, safety, as well as productivity to be analyzed.

In our proposed approach, a sparse 3D geometric scene of the site is reconstructed and progress photographs are geo-registered in a virtual environment. This allows project managers to interactively and remotely browse and explore the as-built scene and geo-registered site construction photographs in a 3D environment. We show from the stand point of progress monitoring how these site photograph logs present an ultimate data set, giving the ability to model a significant portion of as-built geometry at high resolution respective to conditions where enough photographs are being taken. Within the proposed platform, automatic $3 \mathrm{D}$ recognition techniques could be developed to quantify as-built progress from the georegistered images. We present our results on two ongoing construction projects and further discuss technical issues on developing and implementing this new technology for generating and visualizing as-built scenes.

\section{Emerging Field Data Capture Technologies}

For more than a decade, researchers have been pointing out deficiencies in current construction site data collection practices (e.g., manual data collection, need for systematic collection and processing of as-built data to produce useful and real-time progress information (Kiziltas et al. 2008, Navon and Sacks 2007). According to (Navon and Sacks 2007) these research efforts have been motivated by two major drives: (a) an increasing need for real-time feedback and monitoring information and (b) rapid and cost effective technological development in automated data collection technologies for construction. The main technologies designed and implemented in this category are barcode and RFID tags, GPS Systems, Laser scanners and Time-Lapse Photography and Videotaping:

- Barcode and RFID tags have been used to capture and transmit data from a tag embedded or attached to construction components (Kiziltas et al. 2008, Navon and Sacks 2007). Unlike barcodes, RFID tags do not require line-of-sight, close proximity, individual reading and direct contact (Kiziltas et al. 2008). RFIDs and barcodes potentially eliminate non-value adding tasks associated with project management processes, but 
they require frequent installation and maintenance. Additionally they cannot be attached to many types of components and they do not capture progress of partially installed components.

- Laser scanners have been used for construction quality control (Akinci et al. 2006, Jaselkis et al. 2006), condition assessment (Gordon et al. 2004), component tracking (Teizer et al. 2005) and progress monitoring (El-Omari and Moselhi 2008, Bosche and Haas 2008, Su et al. 2006). Although laser scanners are promising in automating data collection, there still is a set of challenges in implementing such technology on construction sites. These limitations include discontinuity of the spatial information, mixed pixel phenomenon (Kiziltas et al. 2008) as well as scanning range and sensor calibration. For example, any moving object in line-of-sight of the scanner would not allow the point cloud of the under-study object to be captured. In addition, the moving object creates additional effort of the user to manually have the point cloud fixed. Also as the laser scanner gets away from the objects, the level of details within the captured components is reduced. Besides laser scanners require regular calibrations as well as warm up time. These limitations are parts of the time consuming process of data collection; however since laser scanners only provide Cartesian coordinate information of the scanned scene, processing such data is time consuming. Also they do not carry any semantic information, such as which point belongs to what structural components. Working with this type of featureless data makes geometric reasoning based on this data tedious and error prone (Kiziltas et al. 2008). Also none of these techniques provide any visual, reliable information about work sequence, site logistics or construction crew. Recently El-Omari and Moselhi (2008) presented a new interesting approach for data collection by combining 3D laser scanners and photogrammetry. The method was shown to be less time-consuming and has higher cost savings compared to single application of laser scanners. Their suggested approach minimizes access limitations of scanner placement, but the processing time required for each scan is still considerably high and the registration of images and 3D point cloud needs further adjustments. Also, laser scanners may not give the possibility of aligning site images taken from arbitrary view points with the 3D point cloud; yet in El-Omari and Moselhi (2008) the common points between laser scanner's 3D point clouds with images have been selected manually. Manual selection of common points between each image and point cloud may make such systems difficult to manage.

- GPS (Geographical Positioning Systems) as a location tracking tool also need line-of-sight between the receiver and the satellite; therefore it cannot normally operate indoors limiting the project context that could be monitored. Behzadan et al. (2008) suggests using WLAN technique as a tracking technique for indoor locations but they also report difficulties in using WLAN set ups on actual construction site and they relate these efficiencies to ongoing works (i.e., changes in soil, structure, plant and equipment, site layout). These inefficiencies necessitate WLAN system to be calibrated after regular intervals to maintain a high level of accuracy. Such regular calibration requirements make the system difficult to manage.

- Time-Lapse Photography and Videotaping: Previous research efforts in using time-lapsed photographs for the purpose of progress tracking goes back to Oglesby et al. (1989) wherein it was reported that application of site photographs allows analysts to focus on the details of the work face while being away from site tensions and confusions and perform time-studies on time-lapsed photographs for productivity improvement. However, lack of advanced technologies for automation had made the process timeconsuming and unattractive to some extent. More recently, Abeid et al. (2003) presented Photo-Net II wherein time-lapse digital movies of construction activities were linked with critical path activities. In PhotoNet II, time-lapse photography was used as a source of spatial as-built information. In addition, GolparvarFard et al. (2007) also recently presented an Augmented Reality (AR) system wherein 3D models are superimposed over time-lapsed photographs.

- Other techniques such as wearable computers (PDAs), speech recognition and touch screens have also helped to collected construction site data electronically (Reinhardt et al. 2000), but current systems still need full time observer(s) to input and process information (Navon and Sacks 2007) and have not minimized the time required to process data.

Also none of these techniques besides (El-Omari and Moselhi 2008) - in which photographs are used to provide more information about the context of the scene - provide visual reliable information about work sequence logistics, site layout or construction crews. Our approach considers all the aspects of as-built data collection: collection, analysis, communication and reporting. We have looked into construction site photo-logs as existing simple yet robust data collection and communication techniques available on all construction sites 
and see how we can effectively use such information to address mentioned problems. In the section that follows, our proposed method of as-built data collection and representation is discussed.

\section{Overview of the Proposed As-Built Representation}

To date, application of unordered daily site photographs for representation of construction progress is almost unexploited. The progress images are usually not organized, uncalibrated, are widely variable and taken under various illumination, resolution, and image qualities. Developing computer vision and image processing techniques that can operate effectively with such imagery is a challenging task. Within such scope, one key challenge is image registration, i.e., gauging correspondences between images, and how they relate to one another in a common 3D coordinate system. This procedure is called Structure from Motion (SfM). While substantial progress has been done in these areas over the last two decades (recent examples: Snavely et al. 2008, Brown and Lowe 2005, Hartley and Zisserman 2004), some challenging aspects are still unresolved. For instance, there is a necessity to work with images that are capturing sites whose appearance is constantly changing due to progress or excessive movement of objects (e.g., site crew and machinery). Also site photographs are sometimes taken only from specific activities under progress or in panoramic fashions. These images may not carry enough information for a more global reconstruction of the scene. Here we present state-of-the-art steps we implemented towards solving this problem (Figure 1):

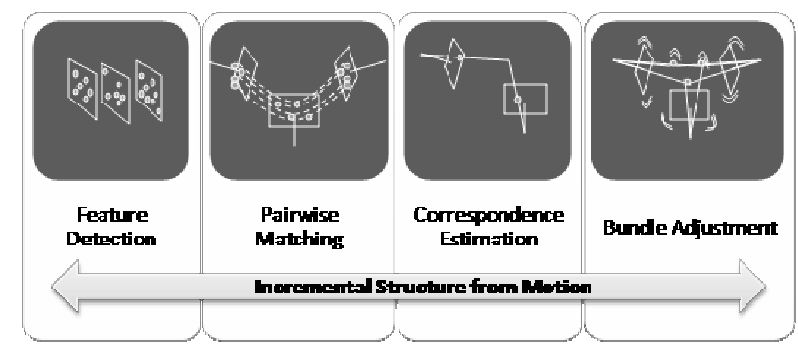

Figure 1. Steps of Structure from Motion from left to right (extended from Nistér and Davidson 2005)

Feature Detection and Correspondence: First step to use site images for reconstruction of the as-built scene is to find distinct features on each image, which allow matching these features across a subset of images. Despite significant research on feature detection and matching, only recently researchers have proposed techniques that prove to be successful in extracting and detecting salient regions (in image/scale space) invariant with respect to scale, orientation and affine transformations. Mikolajczyk et al. (2005) reviews some recently developed view-invariant local image descriptors and experimentally compare their performances.

Structure from Motion (SfM): Aims to reconstruct the unknown 3D structure and estimate camera positions and orientations from a set of image feature correspondences. Photogrammetric techniques such as bundle adjustment (Triggs et al. 1999) are currently used in computer vision for 3D reconstruction and SfM optimization. In our approach, we use the effective method introduced by (Snavely et al. 2007, and, Brown and Lowe 2005). While these techniques have been applied for image navigations and touring, our paper marks the first successful demonstration of SfM technique being applied to geospatial photographs that capture a dynamic construction scene.

Image based Rendering: Following Snavely et al. (2006), image based rendering techniques can be used to synthesize new views of a scene from a set of photographs. In that paper, these techniques are reviewed. Our work is close to Phototour of Snavely et al. (2006) and Sea of Images (Aliaga et al. 2003) where a large set of images is taken throughout architectural spaces. In our work, images are casually acquired on site (as in Snavely et al. 2006), rather than being taken from fixed locations or on guided robot (as in Aliaga et al. 2003).

\section{Discussion on Experimental Setup and Results}

Construction photo-logs for our experiment have been collected on two construction sites on a daily basis. One of the authors has been working on these projects and has taken regular daily construction photos. Rather than only taking photos from specific locations or progress within the day, scenes that capture overall depiction of the construction site are captured as well. Figure 2 shows a variety of photo-logs 
captured for progress analyses in these projects. During the experiment a high-resolution SLR camera was carried. The choice of a high resolution camera was based on the possibility for further enhancement of the algorithm so the quality of the images could be synthetically reduced and the keypoint detection could be tested on synthetically lowered resolution images. To assure the availability of data for further analysis, a larger number of photos than average (about 200/day) have been collected to allow more commonalities between images to be detected. These projects are as follows: (1) Student Dining Hall Project: A two-story masonry and curtain wall LEED Silver certified building with a partial basement of about 139,327sf. This project is a $\$ 36 \mathrm{M}$ steel frame with composite decking in about 25 months of scheduled work. (2) Residence Hall Project: A two-story masonry and curtain wall LEED Silver certified building with a partial basement of about 58,000sf with structure base being reinforced concrete frame. This project is 21 months and the construction cost is approximately $\$ 15 \mathrm{M}$. These projects show two major types of structures which makes them very attractive for our case allowing us to make sure our suggested approach works in different conditions.
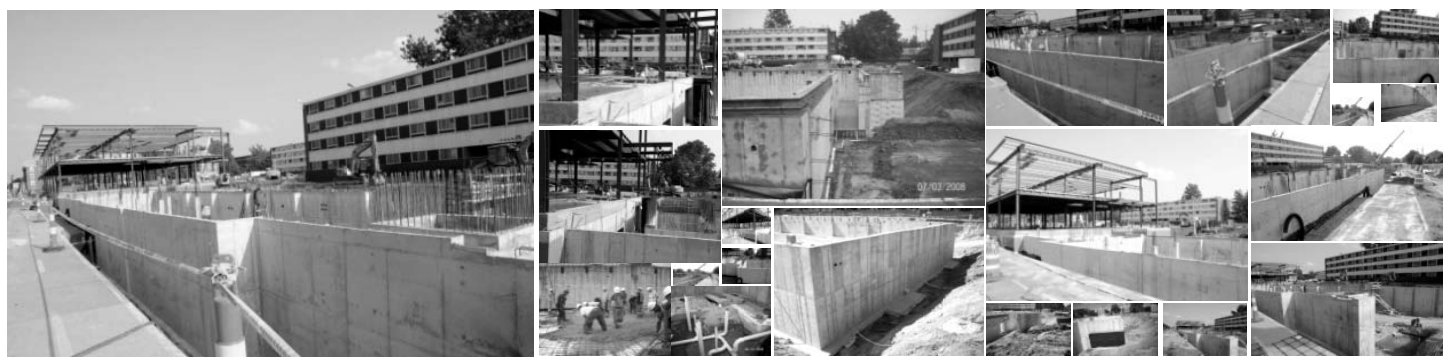

Figure 2. An uncalibrated subset of Student Dining/ Residence Hall Photo-log collected for various site analyses using different cameras on a daily basis

The first step is to find feature points in each image that could be used to estimate the initial structure of the scene. In our work, we use the SIFT keypoint detector (Lowe 2004), because of its good invariance to scale changes and view and illumination transformations as well as its standard application in the computer vision domain (Savarese and Fei-Fei 2007). An image of 3MPixels typically gives about 9,000 to 11,000 features. An example of detected features and number of features detected are illustrated in Figures 3 and 4 respectively.
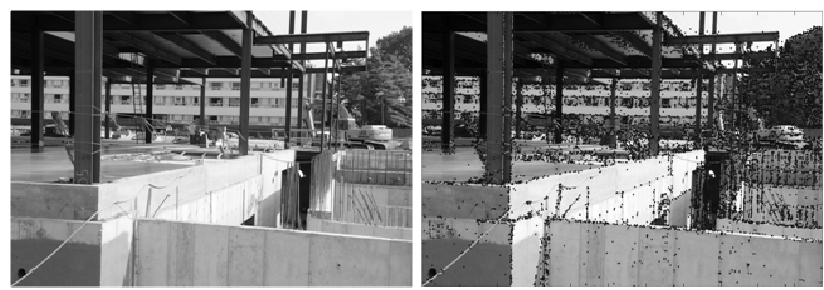

Figure 3. (a) An image taken on 09/27/08 from Student Dining and Residence Hall projects; (b) Same image in grayscale with SIFT feature locations visualized in blue

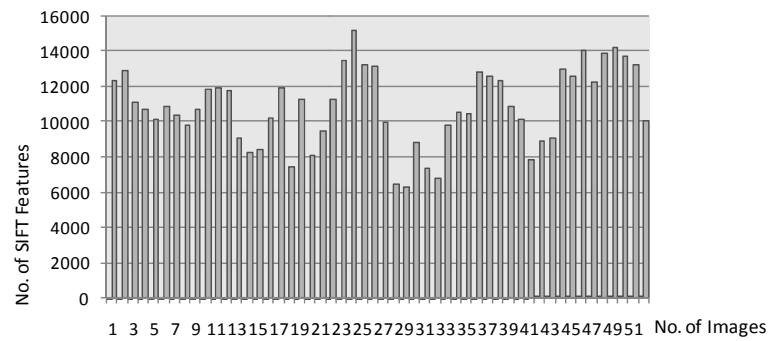

Figure 4. No. of SIFT features on the 52-image subset taken on 09/27/08. No. of SIFT features within the range of $[6481,15160]$. Quality of images synthetically reduced to $25 \%$ of the original form (Tested image dimensions $=2144 \times 1424)$. 
Once the features have been detected over the dataset, we need to detect the number of matching features in each image pair. To minimize computational speed, as experienced by Snavely et al. (2006), we use ANN's priority search algorithm and limit each query to check a limited set. Furthermore, instead of classifying false matches by thresholding the distance to the nearest neighbor, we use the ratio test described by Lowe (2004): for a feature descriptor in image $i$, we find the two nearest neighbors in $j$, with distances $\mathrm{d} 1$ and $\mathrm{d} 2$, then accept the match if $\mathrm{d} 1 / \mathrm{d} 2<0$.6. If more than one feature in $\mathrm{i}$ matches the same feature in $\mathrm{j}$, we remove both of such matches, as one of them is a false match. Figure 5-left shows the number of matched SIFT features within the subset. Due to the sensitivity of reconstruction algorithm to false matches, we use an algorithm to remove such false matches. In our approach, once the matching features are detected in an image pair, we robustly estimate a fundamental matrix for the pair using RANSAC (Fischler and Bolles 1981). The fundamental matrix removes false matches as enforces corresponding features to be consistent under viewpoint transformation. In our model, in each iteration of RANSAC, a fundamental matrix is computed using the 8-point algorithm of Hartley and Zisserman (2004), and then the problem is normalized to improve robustness to noises (See Figure 5-right for refined matched features).

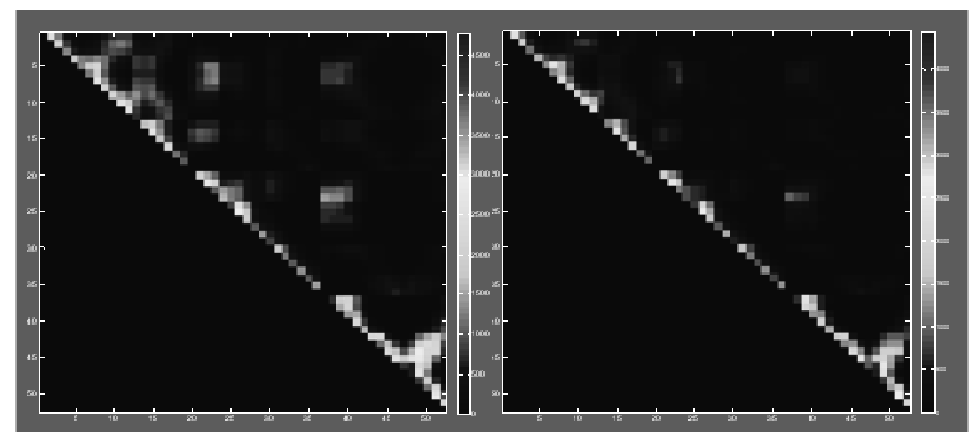

Figure 5. Number of matched SIFT features between each image pair. Both axes show the camera indices and the colored dots visualize the number of SIFT features in image pairs matched before and after the fundamental matrix was fitted to the matching features.

Now, we recover camera extrinsic and intrinsic parameters (extrinsic parameters: rotation, translation; and intrinsic parameters: focal length and distortion) for each image and a 3D location for each keypoint. The recovered parameters should be consistent, in that re-projection error; i.e., sum of distances between the projections of all 3D features and their corresponding image features, is minimized. This minimization problem can be formulated as using the bundle adjustment algorithm (See Triggs et al. 1999 for more details). First, we estimate extrinsic and intrinsic parameters of a single image pair. Since bundle adjustment as other algorithms for solving non-linear problems may get stuck in bad local minima, it is strongly suggested by many researchers (e.g., Snavely et al. 2007, Nistér 2004) to start with a good initial image pair and good estimates for camera parameters in the chosen pair. This initial pair for SfM should have a large number of matches, but also have a large baseline, so that the initial scene can be robustly reconstructed. An image pair that is poorly described by a homographic transformation stratifies this condition. A 2D image homography is a projective transformation that maps points from one image plane to another image plane (Hartley and Zisserman 2004). We find the homography between all image pairs using RANSAC with an outlier threshold of $0.4 \%$ of maximum of image width and height, and store the percentage of feature matches that are inliers to the estimated homography. We select the initial image pair as that with the lowest percentage of inliers to the recovered homography, but with at least 100 matches (As experienced by Snavely et al. 2007). The extrinsic camera parameters for this pair are estimated using Nistér's five point algorithm (Nistér 2004), and then the tracks visible in the image pair are triangulated. A two-frame bundle adjustment for this initial pair is performed. Next, we add another camera to the optimization. We choose the camera that examines the largest number of estimated tracks, and initialize the new camera's extrinsic parameters using the Direct Linear Transform (DLT) technique (Hartley and Zisserman 2004) within a RANSAC procedure. For this RANSAC step, we use an outlier threshold of $0.4 \%$ of maximum of image width or height. We use focal length from the EXIF - exchangeable image file format- tags of JPEG images to initialize the focal length of the new camera and estimate the intrinsic camera matrix (see Snavely et al. 2007 more details). 
Starting from this initial reconstructed scene, we run the bundle adjustment algorithm, allowing new camera and feature points it observes to change while the rest of the model is kept fixed. A feature point is added if it is observed by at least one recovered camera, and if triangulating the location gives a wellconditioned approximation. We estimate the conditioning by considering all pairs of rays that could be used to triangulate that point, and finding the pair of rays with the maximum angle of separation. If this maximum angle is larger than a threshold (As experienced by Snavely et al. 2007) then the point is triangulated. Once the new points have been added, we run another global bundle adjustment to refine the entire as-built reconstructed scene. We use the minimum error solution with the sparse bundle adjustment library of Lourakis and Argyros (2004). This procedure is repeated for all cameras until no remaining camera observes enough reconstructed $3 \mathrm{D}$ points to be reliably reconstructed. Overall only a subset of the used images will be reconstructed. This subset is not selected beforehand, but is determined by the algorithm. After the as-built scene is reconstructed, the scene needs to be used for interactive explorations. Authors implemented an image-based rendering system in Microsoft $\mathrm{C}++$.NET using Microsoft DirectX9 graphics library. The following data structure is used to represent the as-built reconstructed scene: (1) A set of keypoints, in which each keypoint consists of a 3D location and a color that is averaged out from all the progress images that the keypoint is being observed from; (2) A set of cameras, while the extrinsic parameters (translation and rotation), and intrinsic parameters (focal length and distortion in height and width directions) are estimated; and (3) A mapping between each point and all the cameras that observe the point. A list of numbers of cameras which observe the point, the location of the point in local coordinates of the image, and the SIFT keypoint index are all stored. While this information is stored, cameras would be rendered as frusta. Figure 6a \& b show the reconstructed sparse scene from the same image subset and illustrate 6 of the registered cameras. Once a camera is visited in this reconstructed scene, the camera frustum is texture-mapped with a full resolution of the image so user can zoom in and acquire progress and productivity details as well as workspace logistics. Figure $6-\mathrm{c}, \mathrm{d}$, e and $\mathrm{f}$ show the location of a frustum textured while demonstrating how the site image is geo-registered with the as-built point cloud.
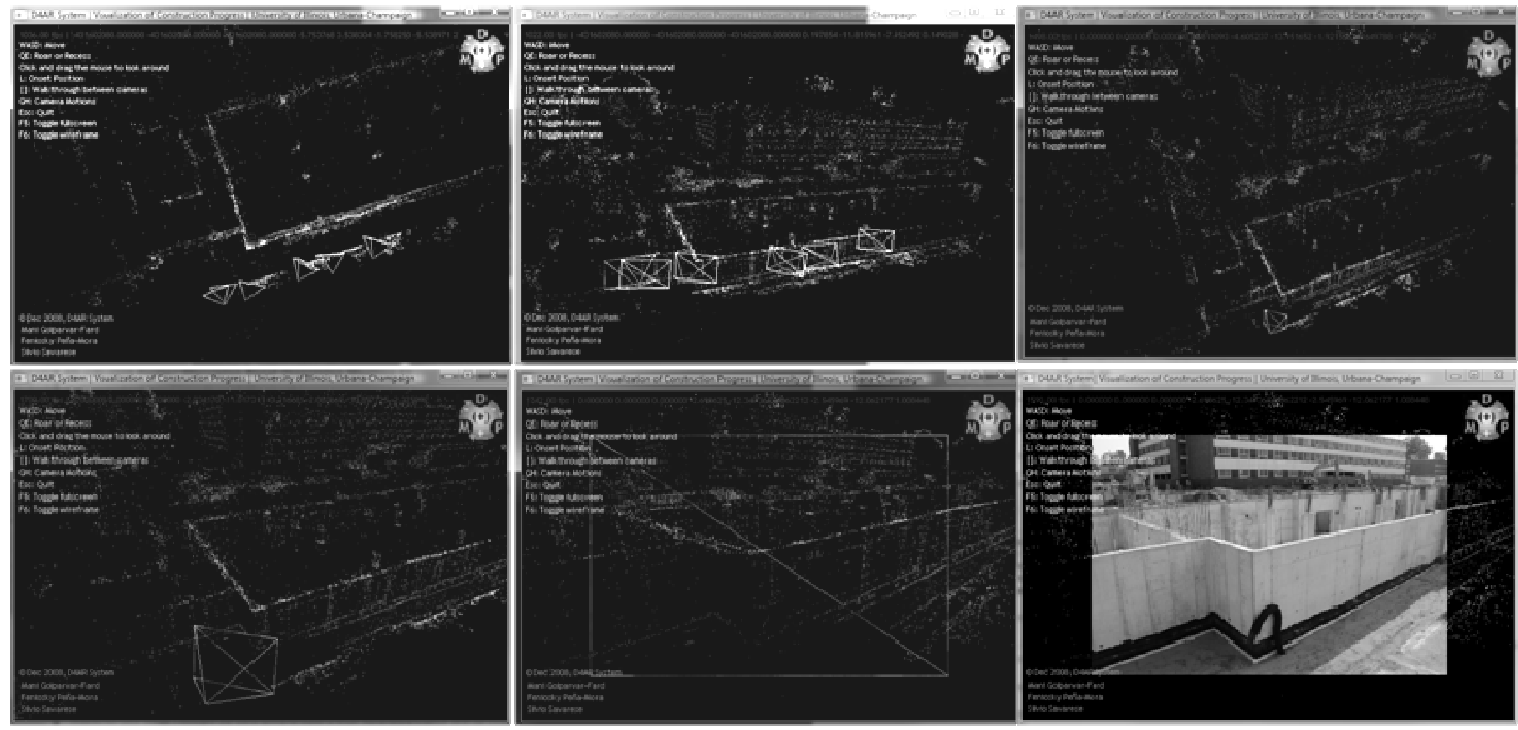

Figure 6. Sparsely reconstructed scene of Residence Hall using 52 images with 25\% of image qualities. Six camera frusta are rendered and geo-registered.

\section{Conclusion}

Our proposed system marks the first system that allows as-built construction spatial information to be visualized using unordered site photo-logs. The demonstrated system has the following benefits: (1) Remote Construction Control Decision Making: It allows project managers, superintendents and other project participants to virtually walk on the construction site, as-of the time the scene has been reconstructed and position themselves in those positions that progress images have been taken. Such an interactive user walkthrough allows progress to be discussed. (2) It minimizes the time required to discuss the as-built scene: Project 
managers and superintendents will spend less amount of time discussing or explaining progress. Rather, they can spend more time on how a control decision could be made. Furthermore, reconstructed as-built scene and geo-registered images allow workspace logistics, safety issues, progress and even productivity of workforce and machinery to be remotely analyzed. Such an as-built system could also be beneficial in weekly contractor coordination meetings, as the workspace could be navigated through the virtual world. (3) Significant cuts in travel time and cost on project executives and architects - Project Executives and architects can study the reconstructed scene and geo-registered images, instead of spending time and money traveling to the jobsite. The reconstructed scene with as-built progress images can be beneficial, especially when the possibility of adding new photographs quickly to the system is considered. Even if a vanishing point of an interest is not registered within the reconstructed scene and is not present in geo-registered image dataset, the user (e.g., owner, project executive) can request the scene to be photographed, since the geo-registration removes confusion on perspective which is inherent in dynamic scenes. Those photographs taken can also be quickly geo-registered which allows a significant problem of progress communication to be resolved. (4) $D^{4}$ AR System- 4 Dimensional Augmented Reality Tool - This system could also be used as a baseline for an Augmented Reality tool wherein as-planned model could be geo-registered within the spatial as-built environment allowing construction progress deviations to be measured, analyzed and communicated. To that extent, authors have proposed D4AR - 4 Dimensional Augmented Reality - system which superimposes the planned model over point cloud and utilizes a traffic light color spectrum for visualizing progress (Golparvar-Fard et al. 2009); (5) Automatic progress tracking- Since this model geo-registers construction site photographs, it could serve as a rich baseline for automating progress monitoring through consistent visual detection of progress deviation and comparison with as-planned information; (6) Registering New Daily Site Photographs- New construction progress photographs can be registered within the system instantly. First, the user can open a set of progress images, and drag and drop each image onto its approximate location on the as-planned model. After each image has been dropped, the system can estimate location, orientation, and focal length of the new image by running the SfM algorithm. First, SIFT keypoints are extracted and matched to the keypoints of the cameras closest to the initial location; then the existing 3D points corresponding to the matches are identified; and finally, these matches are used to refine the pose of the new camera. Our preliminary results show perceived benefits and future potential enhancement of this new technology in construction, in all fronts of automatic data collection, processing and communication; though there are still many technical challenges in developing a full systematic progress monitoring system. These are currently being explored within the research projects highlighted in this paper.

\section{Acknowledgements}

We would like to thank Turner, Williams Brothers, Grunloh Construction companies especially Mr. Greg Cuttell, Mr. Bob Bursack and Mr. Nick Canellis from Turner as well as University of Illinois- Housing, Facilities and Services for their contributions to this project. This work is financially supported by NSF Award No. CMMI-0800500.

\section{References}

[1] Abeid J., Allouche E., Arditi D., and Hayman M. (2003). PHOTO-NET II: a Computer-based Monitoring System Applied to Project Management, J of Automation in Constr, 12 (5), 603-616.

[2] Aliaga D., Funkhouser T., Yanovsky D., and Carlbom I. (2003). Sea of images. IEEE Computer Graphics and Applications, 23 (6), 22-30.

[3] El-Omari S. and Moselhi O. (2008). "Integrating 3D laser scanning and photogrammetry for progress measurement of construction work". J. of Automation in Const. 18 (1), 1-9.

[4] Golparvar-Fard M., Savarese S. and Peña-Mora F. (2009). "Interactive visualization of construction progress monitoring with D4AR - 4 Dimensional Augmented Reality- models.” Proc., Construction Research Congress, Seattle, WA, Apr 2009.

[5] Golparvar Fard, M., Sridharan, A., Lee, S. and Peña-Mora, F. (2007) "Visualization of construction progress monitoring on time-lapsed photograph”, Proc., Constr. Mgmt \& Econ, Reading, UK 
[6] Golparvar Fard M., Staub-French S., Po B. and Tory M. (2006). "Requirements for a mobile interactive workspace to support design development and coordination.” Proc., XI Joint Int. Conf. on Computing in Civil \& Bldg Eng ASCE, Montreal, QC, 3587-3596.

[7] Hartley R. and Zisserman A. (2004). Multiple view geometry, Cambridge Univ. Press, UK.

[8] Jaselskis E., Cackler E., Walters R., Zhang J., and Kaewmoracharoen M. (2006). "Using scanning lasers for real-time pavement thickness measurement”. CTRE Project 05-205, National Concrete Pavement Tech Center, Iowa State Univ.

[9] Kiziltas S., Akinci B., Ergen E. and Tang, P.o (2008). "Technological assessment and process implications of field data capture technologies for construction and facility/infrastructure management", ITcon, 13, Sp. Issue Sensors in Const. and Infrastructure Mgmt., 134-154.

[10] Lourakis M. and Argyros A. (2004). "The design and implementation of a generic sparse bundle adjustment software package based on the Levenberg-Marquardt algorithm” (Technical Report 340). Inst. of Computer Science-FORTH, Heraklion, Crete, Greece.

[11] Lowe D. (2004). "Distinctive image features from scale-invariant keypoints". Int Journal of Computer Vision, Vol. 60, No. 2, 91-110.

[12] Meredith J. and Mantel S. (2003). Project Mgmt: A managerial approach. J. Wiley \& Sons, 5th Ed.

[13] Mikolajczyk K., Tuytelaars T., Schmid C., Zisserman A., Matas J., Schaffalitzky F., Kadir T., and van Gool L. (2005). “A comparison of affine region detectors", Int J. of Computer Vis., 65 (1/2), 43-72.

[14] Navon R. and Sacks R. (2007). "Assessing research in automated project performance control (APPC)." J. of Automation in Construction, 16 (4), 474-484.

[15] Nistér D. (2004). "An efficient solution to the five-point relative pose problem", IEEE Transactions on Pattern Analysis and Machine Intelligence (PAMI), 26 (6), 756-770.

[16] Nistér D. and Davison A. (2005). "Real-Time motion and structure estimation from moving cameras", Tutorial at CVRP 2005.

[17] Oglesby C., Parker H., Howell G. (1989). Productivity improvement in construction, McGraw-Hill.

[18] Poku S. and Arditi D. (2006). "Construction scheduling and progress control using geographical information systems". J. of Computing in Civil Eng., 20 (5), 351-360.

[19] Savarese S. and Fei-Fei L. (2007). :3D generic object categorization, localization and pose estimation". IEEE Intern. Conf. in Computer Vision (ICCV). - Brazil, October, 2007.

[20] Shih N.J., Wu M.C., and Kunz J. (2004). "The inspections of as-built construction records by 3D point clouds". Stanford Univ., CIFE WP \#090.

[21] Snavely N., Seitz S., and Szeliski R. (2006). "Photo tourism: exploring photo collections in 3D." Proc., ACM Transactions on Graphics, 25 (3), 835-846.

[22] Snavely N., Seitz S., and Szeliski R. (2007). "Modeling the world from internet photo collections". Int. J. of Computer Vision.

[23] Snavely N., Seitz S., and Szeliski R. (2008). "Finding path through the world's photos". Proc., ACM Transactions on Graphics, 27 (3), 11-21.

[24] Soibelman L., Wu J., Caldas C., Brilakis I. and Lin K.Y. (2008). "Management and analysis of unstructured construction data types." J. of Advanced Engineering Informatics, 22, 15-27.

[25] Su Y., Hashash Y., and Liu L.Y. (2006). "Integration of construction as-built data via laser scanning with geotechnical monitoring of urban excavation”. J. of Const Eng. Mgmt, 132 (12), 1234-1241.

[26] Teizer J., Kim C., Haas C., Liapi K., and Caldas C. (2005). "Framework for real-time three-dimensional modelling of infrastructure". Geology and Properties of Earth Materials 2005, Transportation Research Board Natl Research Council, Washington, 177-186. 